\title{
ON A CONJECTURE OF E. GRANIRER CONCERNING THE RANGE OF AN INVARIANT MEAN
}

\section{CHING CHOU}

ABstract. The purpose of this paper is to prove the following conjecture of $\mathrm{E}$. Granirer: if $S$ is an infinite right cancellation left amenable semigroup then for each left invariant mean $\phi$ of $S$, $\{\phi(A): A \subset S\}=[0,1]$.

Let $S$ be a semigroup with discrete topology, $m(S)$ the space of bounded real functions on $S$ with the sup norm. $\phi \in m(S) *$ is a mean if $\|\phi\|=1$, and $(\phi, f) \geqq 0$ whenever $f \geqq 0$. A mean $\phi$ is said to be left invariant if $\left(\phi, l_{s} f\right)=(\phi, f)$ for $s \in S$ and $f \in m(S)$, where $l_{s} f \in m(S)$ is defined by $\left(l_{s} f\right)\left(s_{1}\right)=f\left(s s_{1}\right)$. If $m(S)$ has a left invariant mean then we say $S$ is left amenable.

Each mean $\phi$ on $m(S)$ can be considered as a finite additive measure on the family of all subsets of $S$. For $A \subset S, \phi\left(\chi_{A}\right)$ will also be denoted by $\phi(A)$. Clearly, if $\phi$ is a mean then the range of $\phi,\{\phi(A): A \subset S\}$, is a subset of $[0,1]$. The purpose of this paper is to prove the following.

THEOREM. Let $S$ be an infinite right cancellation left amenable semigroup. Then the range of each left invariant mean on $m(S)$ is the whole $[0,1]$ interval.

Granirer stated this theorem as a conjecture in [3]. There he was able to prove it (in a stronger form) for all cases except when $S$ is a socalled "AB group". An infinite torsion group $S$ is an AB group if (a) $S$ is amenable, (b) each infinite subgroup of $S$ is not locally finite, cf. [3].

Each mean $\phi$ on $m(S)$ corresponds to a unique probability measure $\mu_{\phi}$ on $\beta S$, the Stone-Cech compactification of the discrete set $S$. The correspondence is characterized by $(\phi, f)=\int_{\beta S} f-d \mu_{\phi}$, where $f \in m(S)$ and $f^{-}$denotes its continuous extension to $\beta S$. If $B C S, B^{-}$will denote the closure of $B$ in $\beta S$. Sets of the form $B^{-}, B \subset S$, are closed-open in $\beta S$ and they form a topological open basis for $\beta S$.

For each $s \in S$, we have a continuous mapping $s^{\sim}$ of $S$ into $\beta S$ defined by $s^{\sim}\left(s_{1}\right)=s s_{1}, s_{1} \in S$. $s^{\sim}$ has a unique continuous extension to $\beta S$. The extended mapping will also be denoted by $s^{\sim}$. If $S$ is actually a group then, for each $s \in S, s \sim$ is a homeomorphism from $\beta S$ onto $\beta S$ (cf. [1, Lemma 2.1]). Moreover, $\left(s_{1}^{-1} s_{2}\right)^{\sim}=\left(s_{1}^{\tilde{1}}\right)^{-1} s_{\mathbf{2}}$ and $e^{\sim}=$ the iden-

Received by the editors December 12, 1969.

AMS 1969 subject classifications. Primary 4696; Secondary 2875, 5485.

Key words and phrases. Invariant means, range of a measure, amenable groups, Stone-Čech compactification. 
tity function from $\beta S$ onto $\beta S$, where $s_{1}, s_{2} \in S$ and $e$ is the identity of $S$.

LeMma 1. Let $S$ be an infinite group, $w \in \beta S$, and $s_{1}, s_{2} \in S, s_{1} \neq s_{2}$. Then $s_{1} w \neq s_{2} w$.

Proof. By the remark above, we may assume that $s_{1}=e$ and $s_{2}=s \neq e$. By Lemmas 1 and 2 of [2], there exist a positive integer $k$ and subsets $A_{1}, A_{2}, \cdots, A_{k}$ of $S$ such that (1) $\cup_{i=1}^{k} A_{i}=S$, (2) $A_{i} \cap A_{j}=\varnothing$ if $i \neq j$, and (3) $s A_{i} \subset A_{i+1}$ if $i \leqq k-1$ and $s A_{k} \subset A_{1}$. Note that by (1) $\beta S=\cup_{i=1}^{k} A_{i}^{-}$. Thus, if $w \in \beta S$ then $w \in A_{i}^{-}$for some $i$, say, $i=1$. Then, by (3), $s^{\sim} w \in A_{2}^{-}$. Since (2) implies that $A_{1}^{-} \cap A_{2}^{-}=\varnothing$, we conclude $s w \neq w$.

LEMмA 2. Let $S$ be an infinite amenable group. If $\phi$ is a left invariant mean on $m(S)$ then $\mu_{\phi}(\{w\})=0$ for each $w \in \beta S$.

Proof. Let $w \in \beta S$. Choose a subset $\left\{s_{1}, \cdots, s_{n}\right\}$ of $S$, where $s_{i} \neq s_{j}$, if $i \neq j$. Then, by Lemma $1, s_{i} w \neq s_{j} w$ if $i \neq j$. It is clear that we can choose a closed-open neighborhood $A^{-}$of $w$ such that $s_{i} A^{-} \cap s_{j} A^{-}=\varnothing$ if $i \neq j$. Denote the characteristic function of $A$ in $S$ by $\chi_{A}$. Then

$$
\begin{aligned}
1 & =\mu_{\phi}(\beta S) \geqq \sum_{i=1}^{n} \mu_{\phi}\left(\tilde{s_{i}} A^{-}\right) \\
& =\sum_{i=1}^{n} \mu_{\phi}\left(\left(s_{i} A\right)^{-}\right) \\
& =\sum_{i=1}^{n} \phi\left(s_{i} A\right)=\sum_{i=1}^{n} \phi\left(l_{-1} \chi_{s_{i}}\right) \\
& =n \phi(A)=n \mu_{\phi}\left(A^{-}\right) \\
& \geqq n \mu_{\phi}(\{w\}) .
\end{aligned}
$$

Since $n$ can be arbitrarily big, $\mu_{\phi}(\{w\})=0$.

LEMMA 3. Let $X$ be an infinite discrete set and $\phi$ be a mean on $m(X)$ such that $\mu_{\phi}(\{w\})=0$ for each $w \in \beta X$. Then $\{\phi(A): A \subset X\}=[0,1]$.

Proof. Note first that $\mu_{\phi}$ is nonatomic. Indeed, if $\mu_{\phi}$ has atoms then there is a compact atom $K$. We may cover $K$ by a finite number of open sets $U_{1}, \cdots, U_{n}$ with $\mu_{\phi}\left(U_{i}\right)<\mu_{\phi}(K)$ for $i=1,2, \cdots, n$. Since $K$ is an atom, $\mu_{\phi}\left(U_{i} \cap K\right)=0$, and hence, $\mu_{\phi}(K)=0$, a contradiction. Consequently, $\mu_{\phi}$ is nonatomic and by Liapounoff's convexity theorem, cf. [4], ${ }^{1}\left\{\mu_{\phi}(\Omega): \Omega\right.$ runs over Borel subsets of $\left.\beta X\right\}=[0,1]$.

${ }^{1}$ Many thanks are due to R. Bourgin for referring this paper to us. 
Let $\Omega$, a Borel subset of $\beta X$, and $\epsilon>0$ be given. Since $\mu_{\phi}$ is regular, there exist a closed set $\Gamma$ and an open set $\Lambda$ of $\beta X$ such that $\Lambda \supset \Omega \supset \Gamma$ and $u_{\phi}(\Lambda \backslash \Gamma)<\epsilon$. Since $\Gamma$ is compact, we can find a closed-open subset $A^{-} \subset \beta X$ such that $\Lambda \supset A^{-} \supset \Gamma$, and hence, $\left|\mu_{\phi}\left(A^{-}\right)-\mu_{\phi}(\Omega)\right|<\epsilon$. Thus we conclude that $\{\phi(A): A \subset X\}$ is dense in $[0,1]$.

Let $\lambda \in(0,1)$ be given. Choose a sequence $\left(\lambda_{n}\right)$ in $(0,1)$ such that $\lambda_{2 n-1}>\lambda_{2 n+1}>\lambda>\lambda_{2 n+2}>\lambda_{2 n}, n=1,2, \cdots$, and $\lim _{n} \lambda_{2 n-1}=\lambda=\lim _{n} \lambda_{2 n}$. Since $\{\phi(A): A \subset X\}$ is dense in $[0,1]$, we can choose a set $A_{1} \subset X$ such that $\lambda_{3}<\phi\left(A_{1}\right)<\lambda_{1}$. Similarly, since $\left\{\phi(A): A \subset A_{1}\right\}$ is dense in $\left[0, \phi\left(A_{1}\right)\right]$, there exists $A_{2} \subset A_{1}$ such that $\lambda_{2}<\phi\left(A_{2}\right)<\lambda_{4}$. Again, since $\left\{\phi(A): A \subset A_{1} \backslash A_{2}\right\}$ is dense in $\left[0, \phi\left(A_{1} \backslash A_{2}\right)\right]$, we can choose $B_{3} \subset$ $A_{1} \backslash A_{2}$ such that $\lambda_{5}<\phi\left(A_{2} \cup B_{3}\right)<\lambda_{3}$. Set $A_{3}=A_{2} \cup B_{3}$. Continue this process. We get a sequence of subsets $\left(A_{n}\right)$ of $X$ such that

$$
A_{2 n-1} \supset A_{2 n+1} \supset A_{2 n+2} \supset A_{2 n}
$$

and

$$
\lambda_{2 n+1}<\phi\left(A_{2 n-1}\right)<\lambda_{2 n-1}, \quad \lambda_{2 n}<\phi\left(A_{2 n}\right)<\lambda_{2 n+2},
$$

for $n=1,2, \cdots$ Let $A=\bigcap_{n=1}^{\infty} A_{2 n-1}$. Then $A_{2 n-1} \supset A \supset A_{2 n}$, and hence, $\lambda_{2 n-1}>\phi(A)>\lambda_{2 n}, n=1,2, \cdots$. Thus $\phi(A)=\lim _{n} \lambda_{n}=\lambda$ and the proof is completed.

Proof of The Theorem. By Theorem 1 and Lemma 4 of [3], we may assume that $S$ is an infinite amenable group. The Theorem is then a direct consequence of Lemma 2 and Lemma 3.

\section{REFERENCES}

1. C. Chou, On the size of the set of left invariant means on a semigroup, Proc. Amer. Math. Soc. 23 (1969), 199-205.

2. E. Granirer, Extremely amenable semigroups, Math. Scand. 17 (1965), 177-197. MR 33 \#5760.

3. - On the range of an invariant mean, Trans. Amer. Math. Soc. 125 (1966), 384-394. MR 34 \#4390.

4. J. Lindenstrauss, $A$ short proof of Liapounoff's convexity theorem, J. Math. Mech. 15 (1966), 971-972. MR $34 \sharp 7754$.

State University of New York at Buffalo, Amherst, New York 14226 